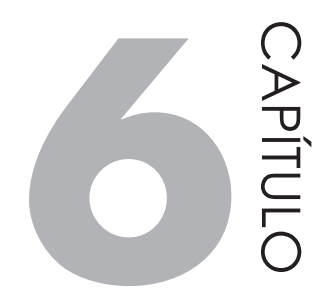

\title{
EQUAÇÃO DA CONTINUIDADE QUADRIDIMENSIONAL
}

Da equação diferencial da continuidade Seção 5.9 .1 temos: $\operatorname{div} \vec{j}+\frac{\partial \rho}{\partial t}=0$, sendo $\vec{j}=\rho \vec{v}$, a densidade de corrente.

Multiplicando e dividindo $\frac{\partial \rho}{\partial t}$ por $\mathrm{v}_{0}$, obtemos: $\frac{v_{0}}{v_{0}} \frac{\partial \rho}{\partial t}=\frac{\partial \rho v_{0}}{\partial t} \frac{\partial t}{\partial x_{0}}=\frac{\partial j_{0}}{\partial x_{0}}$

$$
\text { Pois, } j_{i}=\rho v_{i}=\rho \frac{d x_{i}}{d t} \quad \text { e } \quad j_{1,2,3}=j_{x, y, z} \quad j_{0}=i c \rho^{1} \quad \therefore v_{0}=i c
$$

Portanto, essa última equação tridimensional torna-se quadridimensional: $\frac{\partial j_{i}}{\partial x_{i}}=0 \operatorname{com} x_{0}$ sendo a $4^{\mathrm{a}}$ dimensão.

Assim, conclui-se que o regime é permanente, quadridimensionalmente falando, e a carga é conservada no hipervolume $d \Omega=d V d t$, independentemente de seu tamanho; elementar ou não, infinitesimal ou não, porém no espaço quadridimensional. Também significa que a carga se conserva no espaço-tempo.

(LANDAU, RAINICH, SOKOLNIKOFF)

Ver Seção 5.25, Equação (5.6b), $x_{0}=c t i, v_{0}=\frac{\partial x_{0}}{\partial t}=i c$. Como $i$ e $c$ são constantes, $v_{0}$ é constante. 
De fato: $\int \frac{\partial j_{i}}{\partial x_{i}} d \Omega=0 \Rightarrow \int \frac{\partial}{\partial x_{i}} \rho \frac{d x_{i}}{d t} d V d t=0 \Rightarrow \int d \rho d V=0 \quad \therefore \rho d V=0$

Assim: como $\rho=\frac{d Q}{d V} \quad \therefore \frac{d Q}{d V} d V=0, \log \mathrm{O}: d Q=0 \quad \therefore Q=$ cte

Ver Seção 5.9, Equação (0).

Veja-se uma demonstração diferente de Landau e Lifchitz em teoria do campo. Só que nossa demonstração é bem mais simples.

Pode-se fazer o mesmo raciocínio para a massa. Entretanto, deve-se levar em conta a equivalência entre massa e energia, como foi demonstrado por Einstein, como veremos no capítulo $7: E=c^{2} \Delta \mathrm{m}$, secções 7.8 e 7.15 . 